\title{
Laparoscopic repair using an endoscopic linear stapler for management of iatrogenic colonic perforation during screening colonoscopy
}

\author{
Tae Woon Kim, Hyung Ook Kim, Kyung Uk Jung, Sung Ryol Lee \\ Department of Surgery, Kangbuk Samsung Hospital, School of Medicine, Sungkyunkwan University, Seoul, South Korea
}

Videosurgery Miniinv 2019; 14 (2): 216-222

DOI: https://doi.org/10.5114/wiitm.2018.77719

\begin{abstract}
Introduction: Colonoscopy is a safe and effective procedure, but it is also an inevitably invasive one. Laparoscopic repair of colonoscopic perforations has been reported to be a safe and effective treatment.

Aim: We present our surgical technique and outcomes of laparoscopic repairs using an endoscopic linear stapler for iatrogenic colonic perforation during screening colonoscopy.

Material and methods: Laparoscopic repair using an endoscopic linear stapler for iatrogenic colonic perforation during screening colonoscopy was performed by two experienced laparoscopic surgeons on 14 consecutive patients between April 2010 and December 2017 at our hospital. Using prospectively collected data, an observational study was performed on a per protocol basis.

Results: The mean age of the 14 patients who underwent laparoscopic repair was $56.6 \pm 9.1$ years. The most common perforation site was the sigmoid colon in 10 (71.4\%) patients, followed by the rectosigmoid junction in 3 (21.4\%) patients and the splenic flexure in 1 (7.1\%) patient. The median perforation size was 10 (range: 5-30) mm. The mean operation time was $73.9 \pm 28.2$ min. Postoperative complications occurred in 1 (7.1\%) patient. There was no postoperative mortality or reoperation within 30 days after surgery. The median time to tolerance of a regular diet was 5 (range: 3-6) days. The median postoperative hospital stay was 8.5 (range: 5-15) days.

Conclusions: Laparoscopic repair using an endoscopic linear stapler is a safe, easy, and effective surgical technique to treat colonic perforation related to screening colonoscopy.
\end{abstract}

Key words: laparoscopy, colonoscopy, surgical stapler, colon perforation.

\section{Introduction}

Colonoscopy is a safe and effective method for diagnosis and treatment of benign or malignant colorectal diseases, but it is also inevitably an invasive procedure [1]. Among other serious complications that may occur during colonoscopy, the reported incidence of perforation ranges from $0.06 \%$ to $0.12 \%$ [2-4]. In the last decade, the overall rate of colonoscopic perforation has been less than $0.1 \%$ [5].
Although nonoperative management may be feasible and beneficial in some patients [6-8], urgent surgical intervention should be considered first in most cases of colonoscopic perforation. Although the incidence of colonoscopic perforation is very low, this may be the most frustrating experience for an endoscopist, especially if it occurs during a screening colonoscopy. Minimally invasive surgery could allow both patients and endoscopists to experience less physical and emotional trauma following 
this occurrence. Laparoscopic techniques have been used in many cases of acute abdominal disease, and have shown good results [9].

After the laparoscopic repair of colonoscopic perforation was first described [10], many laparoscopic treatments were reported [11-18]. Laparoscopic direct suture can be performed for the management of small perforations without extensive tissue injury, but some larger or extensive cases may need a tangential resection or segmental resection [14] A couple of reports on the primary repair of colonoscopic perforations have described the performance of a tangential resection using an endoscopic linear stapler [10, 11, 14, 15].

Laparoscopic repair using an endoscopic linear stapler may be safe, easy, and rapid, even in cases of larger injuries, since this method does not require the use of laparoscopic suturing and knot-tying techniques. However, several concerns keep this endoscopic linear stapler repair technique from being widely used, including the following: (1) the margin of the perforation could be injured by the laparoscopic instrument; (2) larger tangential resection might lead to postoperative colonic stenosis; and (3) the endoscopic linear stapler should be applied only in a transverse manner along the running direction of the colon to avoid postoperative colonic stenosis and, thus, cannot necessarily be used in all instances of colonoscopic perforation [15].

\section{Aim}

Herein, we present our surgical technique and the outcomes of laparoscopic repair using an endoscopic linear stapler for iatrogenic colonic perforation during screening colonoscopy.

\section{Material and methods}

This study involved 15 consecutive procedures performed by one of two experienced laparoscopic colon and rectal surgeons included among the surgical on-call team between April 2010 and December 2017 at Kangbuk Samsung Hospital, Sungkyunkwan University School of Medicine, Seoul, South Korea. Any surgery for perforation occurring after a therapeutic colonoscopy was excluded from this study. This study was approved by the ethics committee at our institution (KBSMC 2017-12-027). Using prospectively collected data, an observational study was performed on a per protocol basis.

\section{Surgical procedure}

After general anesthesia, all patients were placed in the modified lithotomy position. In all cases, a to-

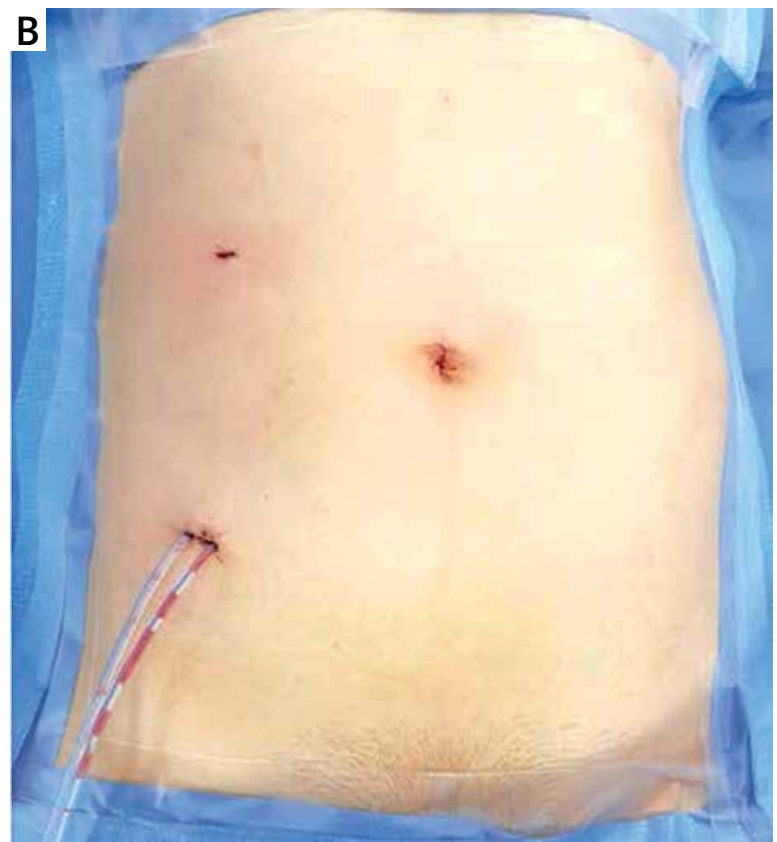

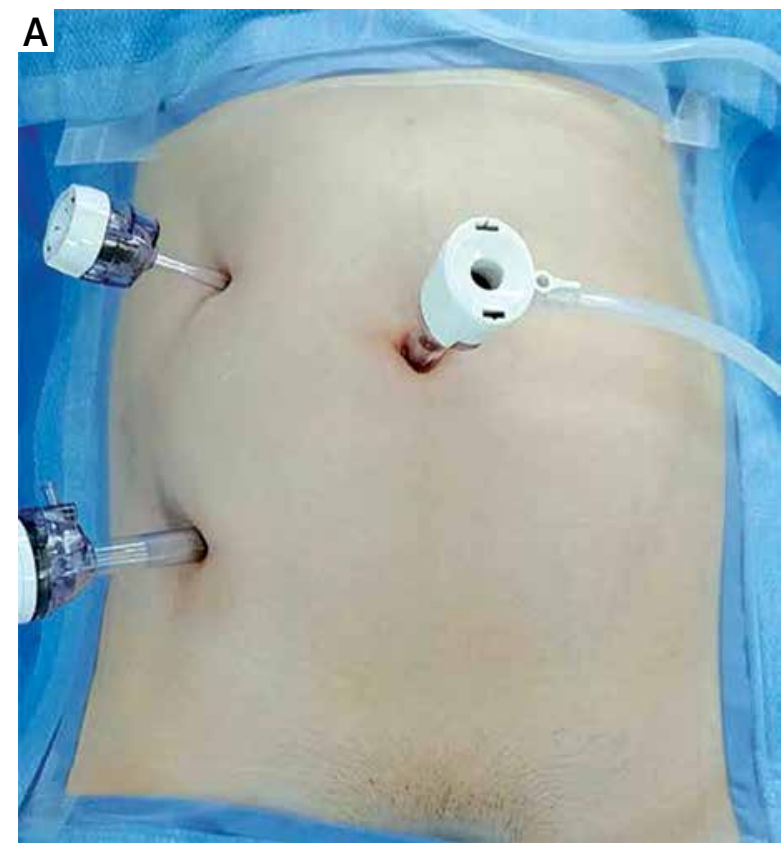

Photo 1. Laparoscopic repair for colonic perforation in a 38-year-old male patient. After trocar insertion (A) and immediate postoperative wound and drain (B) 
tal of three trocars were inserted (Photo 1). Using a modified Hasson technique to access the peritoneum, a $12 \mathrm{~mm}$ blunt laparoscopic trocar was inserted into the supraumbilical position including the center of the umbilicus. One additional $12 \mathrm{~mm}$ laparoscopic trocar was inserted into the right iliac fossa position (the right lower quadrant) under laparoscopic direct vision. The other $5 \mathrm{~mm}$ trocar was inserted into the right upper quadrant. Intraperitoneal exploration was performed to find the perforated area, which was expected to be located in a defined place based on the preoperative computed tomography (CT) and colonoscopic findings. Once the perforated area was found, the pericolic adipose tissue around the defect was removed to expose the margin of the defect using a laparoscopic ultrasonic shear. If the perforation was on the mesenteric margin, there were air bubbles in the mesentery or pericolic fat, where the perforation site could be found after pericolic adipose tissue was removed using a laparoscopic ultrasonic shear. The size of the perforation was determined based on the intraoperative findings via a comparison with the jaw length of the laparoscopic grasper. The margin of the defect was lifted gently using one $5 \mathrm{~mm}$ atraumatic grasper. A $60 \mathrm{~mm}$ articulated endoscopic linear stapler (Purple; height-progressive rows of $3.0 \mathrm{~mm}, 3.5 \mathrm{~mm}$ and $4.0 \mathrm{~mm}$, Endo GIA Reload with Tri-Staple Technology, Covidien) was introduced through a $12 \mathrm{~mm}$ right lower quadrant trocar. The jaw of the endoscopic stapler was placed across the defect, as transversely as possible, although it was deemed the use of an oblique position was not necessarily bad, and stapling was carried out. After complete tangential resection including the defect, the resected piece of colon was removed via a $12 \mathrm{~mm}$ right lower quadrant trocar (Photo 2). The specimen from tangential resection was checked to confirm whether the perforation site was completely
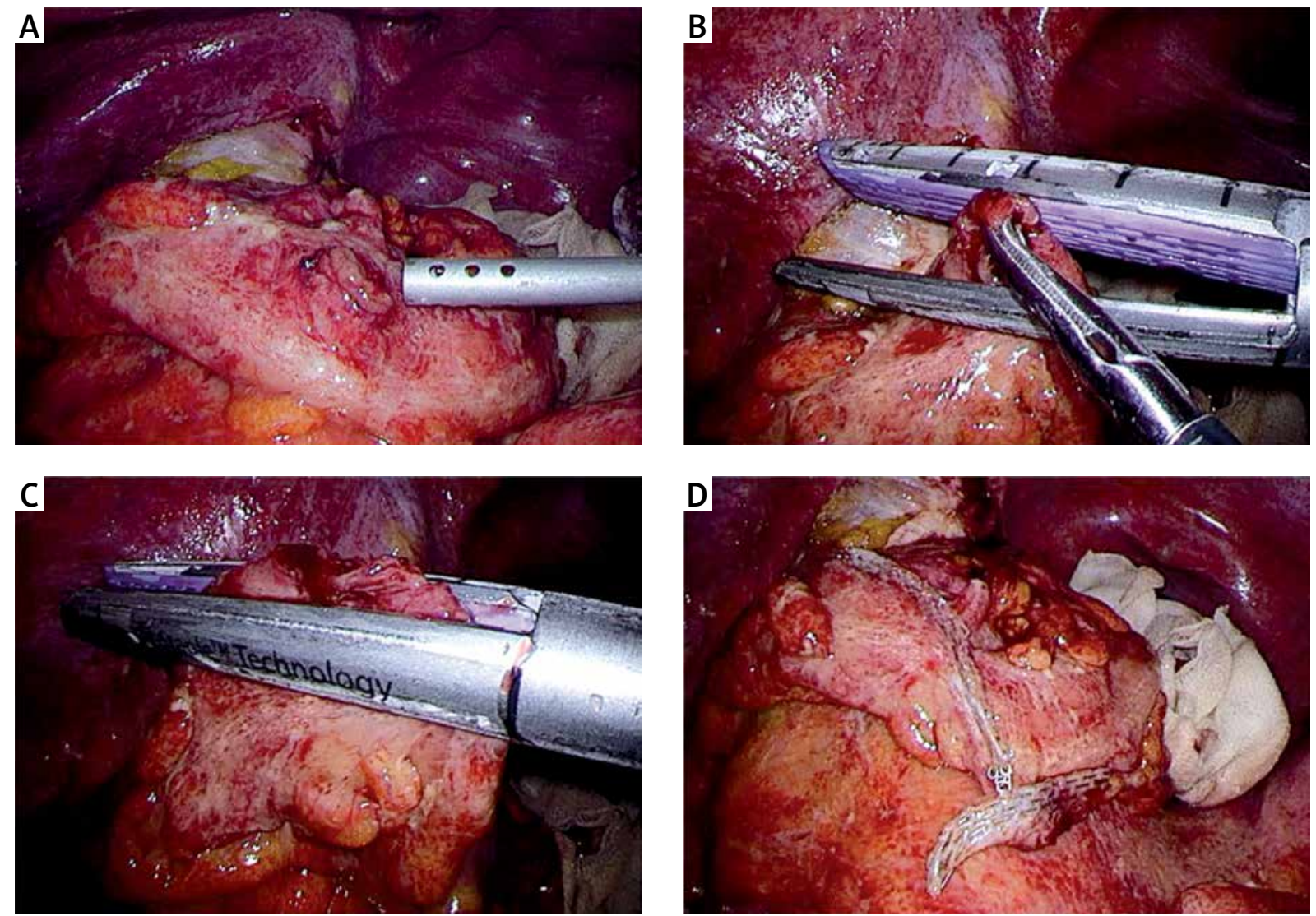

Photo 2. A $10 \mathrm{~mm}$ sized perforation at the sigmoid colon (A) in a 63-year-old male patient who underwent primary closure using a laparoscopic linear stapler after mobilization of the sigmoid colon (B, C). Oblique tangential resection was performed at the site of perforation along the running direction of the sigmoid colon (D) 
included. No other leakage test was performed. In one case presenting with a larger defect $(30 \mathrm{~mm})$, two passing sutures were performed without knot-tying to lift up into balance the margin of the perforation, and then a tangential resection was performed using the endoscopic stapler. Only one $60 \mathrm{~mm}$ stapler was sufficient to repair the colonic perforation in all cases. After a peritoneal toilet was performed, two lines of closed suction drains were inserted into the $12 \mathrm{~mm}$ trocar site (Photo 1 ).

\section{Perioperative management}

In all patients, colonic perforation was confirmed by the presence of free air on an abdominal CT taken after the colonoscopy. All patients received intravenous antibiotics after diagnosis, specifically, third-generation cephalosporin and metronidazole. A urinary catheter was inserted in the operating room following general anesthesia. All anesthetic procedures were performed in a routine fashion. Intravenous patient-controlled analgesia was postoperatively employed for pain relief according to patient preference. The urinary catheter was removed at 6 am on the first postoperative day, after which all patients were encouraged to ambulate. A clear liquid diet was started after bowel sound presented and signs of peritonitis improved, and patients were advanced to a regular diet when a full liquid diet was tolerated or flatus was observed. Patients were discharged from the hospital to home after they demonstrated tolerance of a regular diet and experienced a normal bowel movement.

\section{Results}

\section{Patient characteristics}

In total, 14 patients (with a mean age of $56.6 \pm 9.1$ years) in whom laparoscopic repair was technically completed were analyzed. One patient was converted to open surgery because of a severe peritoneal adhesion resulting from a previous total abdominal hysterectomy; thus, she was ultimately excluded from this study. One patient underwent screening colonoscopy in our institution, while the other 13 patients underwent the procedure at outside institutions. Clinical data, including gender, American Society of Anesthesiologists (ASA) score, history of previous major abdominal surgery, and the site and size of the perforation are shown in Table I. There was no patient who had concomitant colonic tumor. Categorical data are presented as percentages and quantitative data as means \pm standard deviations or median values with ranges.

\section{Perioperative outcomes}

Altogether, 14 patients underwent laparoscopic repair using an endoscopic linear stapler at a median interval of $6 \mathrm{~h}$ after perforation during screening colonoscopy. The most delayed patient underwent the surgery $10 \mathrm{~h}$ after colonoscopy; he is the patient presented in Photo 2 and is one of the patients who underwent colonoscopy at an outside institution. He underwent colonoscopy again 34 months after surgery because of hematochezia, which was confirmed to be due to bleeding from internal hemorrhoids. A scar from the linear stapling was observed, but there was no colonic stenosis (Photo 3).

The perforation sites were the sigmoid colon in $10(71.4 \%)$ patients, the rectosigmoid junction in $3(21.4 \%)$, and the splenic flexure in 1 (7.1\%). The median perforation size was 10 (range: $5-30) \mathrm{mm}$. The mean operation time was $73.9 \pm 28.2 \mathrm{~min}$ (Tables I and II).

Naturally, in all cases there was fecal peritonitis to some degree, but no solid fecal peritonitis, since

Table I. Clinical characteristics and operative findings

\begin{tabular}{|lc|}
\hline Parameter & $\begin{array}{c}\text { Number of patients } \\
(n=14)\end{array}$ \\
\hline Age [years] & $56.6 \pm 9.1$ \\
\hline Gender (males/females) & $6 / 8$ \\
\hline ASA score, $n$ (\%): & $4(28.6)$ \\
\hline 1 & $9(64.3)$ \\
\hline 2 & $1(7.1)$ \\
\hline 4 & $0(0)$ \\
\hline $\begin{array}{l}\text { History of previous abdominal } \\
\text { surgery, } n \text { (\%) }\end{array}$ & $10(35.7)$ \\
\hline \begin{tabular}{l} 
Site of perforation, $n$ (\%): \\
\hline Sigmoid colon
\end{tabular} & $10(5-30)$ \\
\hline Size of perforation [mm] & $3(21.4)$ \\
\hline
\end{tabular}

Values are presented as number (\%) and mean value \pm standard deviation or median value (range). ASA - American Society of Anesthesiologists. 

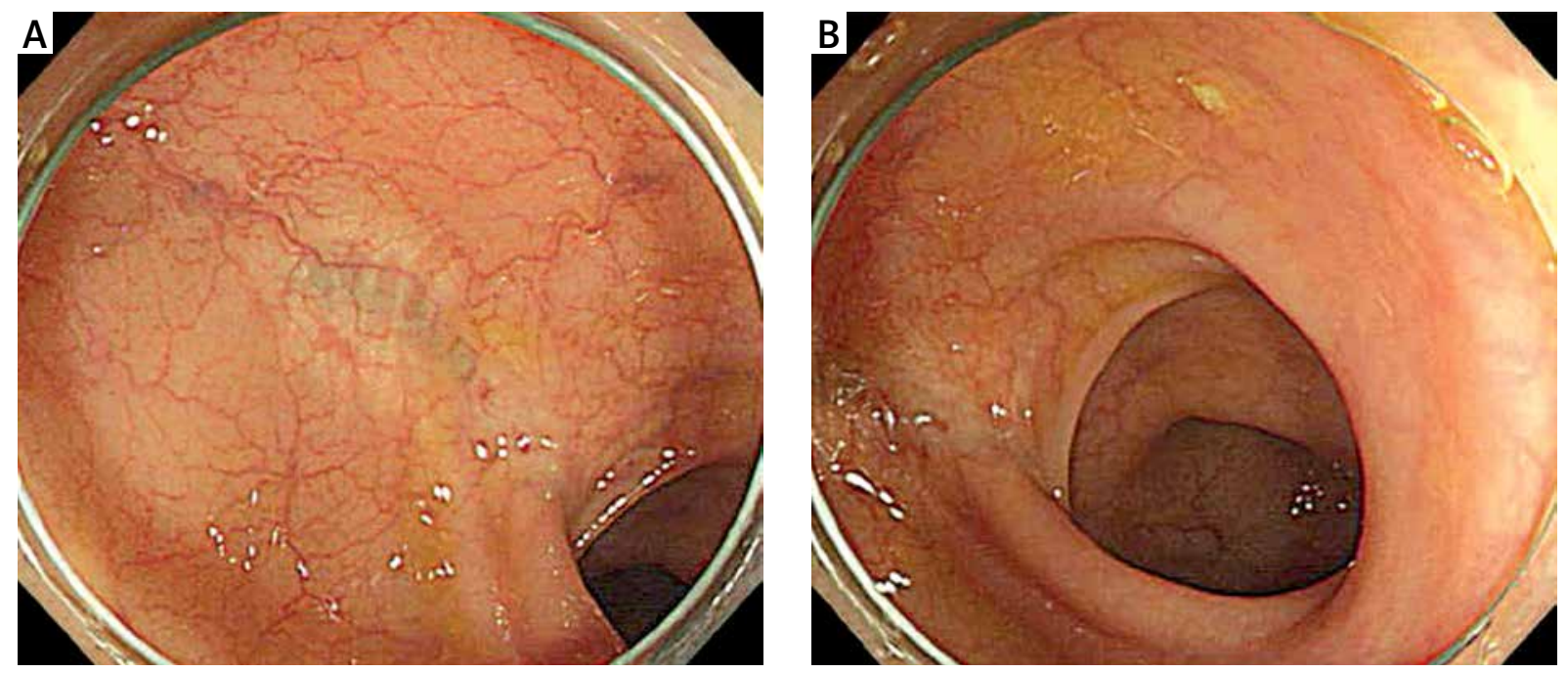

Photo 3. Colonoscopic findings of the patient in Photo 2 after 34 months. Note the complete healing (A) and no colonic stenosis (B)

Table II. Perioperative outcomes and postoperative recovery

\begin{tabular}{|lc|}
\hline Parameter & $\begin{array}{c}\text { Number of } \\
\text { patients }(n=14)\end{array}$ \\
\hline Operation time [min]: & $73.9 \pm 28.2$ \\
\hline Mean & $65(40-135)$ \\
\hline Median & $1(7.1)$ \\
\hline Postoperative complications (\%) & $1(7.1)$ \\
\hline Reoperation within 30 days of surgery & $0(0)$ \\
\hline 30-day postoperative mortality & $0(0)$ \\
\hline Time to tolerance of regular diet [days] & $5(3-6)$ \\
\hline Postoperative hospital stay [days] & $8.5(5-15)$ \\
\hline
\end{tabular}

Values are presented as number (\%) and mean value \pm standard deviation or median value (range).

the colonic perforation occurred in a colon that has been mechanically well prepared. Therefore, fecal peritonitis could be managed by peritoneal toilet and surgical drain without defunctioning enterostomy.

One $(7.1 \%)$ patient experienced a postoperative complication of wound infection at the umbilical trocar site. There was no postoperative mortality or reoperation within 30 days after surgery. The median time to tolerance of a regular diet was 5 (range: 3-6) days. The median postoperative hospital stay was 8.5 (range: 5-15) days (Table II).

\section{Discussion}

latrogenic perforation during screening colonoscopy may be the most frustrating experience for both the patient and the endoscopist involved. Laparoscopic repair may offer a way to alleviate suffering. Unfortunately, a lack of experience with laparoscopic surgery and insufficient laparoscopic techniques often lead to the use of open surgery to repair the colon perforation [7]. The present study demonstrates that laparoscopic repair using an endoscopic linear stapler for iatrogenic colonic perforation during screening colonoscopy is a safe and easily performed technique.

Colonic perforation during diagnostic colonoscopy may result from mechanical forces (shear and/or stretch pressure from the scope) against the bowel wall [19]. Therefore, regions with acute angles and redundancy are the most frequent sites of colonoscopic perforation. As in our study, the most common perforation sites during colonoscopy are typically the sigmoid and rectosigmoid colon $[17,18,20]$. Of course, the redundant sigmoid colon would be the most suitable site for laparoscopic application of an endoscopic linear stapler.

A couple of concerns exist regarding the performance of laparoscopic repair using an endoscopic linear stapler. For example, the margin of perforation could be injured by the laparoscopic instrument [21]. However, in our study, a commercially available atraumatic grasper did not incite macroscopic injury in the colonic tissue in the margin of perforation, 
which is what often leads to the need for a larger resection or additional laparoscopic suturing.

Notably, a larger tangential resection of the colon might lead to postoperative colonic stenosis. Typically, however, the margin of the defect was easily lifted by one $5 \mathrm{~mm}$ atraumatic grasper and the tangential resection was not so large as to lead to postoperative colonic stenosis. In our study, only one larger defect $(30 \mathrm{~mm})$ needed two passing sutures without knot-tying to lift up into balance the margin of the perforation. Lifting of the perforated colonic margin using passing sutures is effective because stapling could cover all layers and the limited resection would lessen the possibility of postoperative colonic stenosis, even in cases of larger defects [10, 15].

Additionally, although it is recommended that only transverse stapling along the running direction of the colon be performed to avoid postoperative colonic stenosis, oblique stapling also did not lead to stenosis in this study, as presented in Photos 2 and 3. It does not seem necessary to insert an additional $12 \mathrm{~mm}$ trocar to make the jaw of the stapler sit transversely across the defect. So far, to our knowledge, no other study performed on this subject has reported colonic stenosis after a stapling repair for a perforated colon.

Our operation time was similar to that reported previously for laparoscopic repair using an endoscopic linear stapler for colonoscopic perforation [15]. A couple of reports using laparoscopic direct suture showed a longer operation time [16, 18]. Laparoscopic stapling might reduce the operation time, since it does not necessitate laparoscopic suturing and knot-tying techniques. The majority of the operation time was used for the peritoneal toilet in our cases. A shorter operation time could still allow for sufficient time to establish the peritoneal toilet. Furthermore, the stapling technique could minimize the handling of the perforated colon, which can lead to spilling out of the colonic content into the peritoneal cavity.

Nonoperative management might be feasible and beneficial in certain patients [6-8], and endoscopic repairs using clips or sutures have been reported as a method to avoid the additional trauma associated with open or laparoscopic surgery [20, 22-26]. The technical success rate for endoscopic clip closure for iatrogenic colon perforation in selected patients was $90.6 \%$ to $96.4 \%$, but, after endoscopic clip closure, treatment failure, which required a surgical approach to correct, occurred in $7.4 \%$ to $24.1 \%$ of patients [20, 22, 24]. Although closure of a large ( $10 \mathrm{~mm}$ ) colonoscopic perforation with endoscopic clips is also a viable therapeutic option, there has not been enough evidence on this concept until now [25]. The important thing to note is that urgent surgical intervention should be considered first in most cases of colonoscopic perforation.

In the present study, there was no postoperative mortality and only one minor postoperative complication. This study enrolled patients who experienced iatrogenic colonic perforation during screening colonoscopy. A screening colonoscopy is a diagnostic method performed on an asymptomatic person to test for the presence of colorectal cancer or colorectal polyps. In Korea, screening colonoscopy is performed in all people who have a positive result on a stool occult blood test enforced by the national health insurance; a screening test including a free colonoscopy is offered annually to people over the age of 50. Most individuals who undergo screening colonoscopy experience no serious medical problems: only $1(7.1 \%)$ patient had an ASA score of 3 in this study. Furthermore, the perforations that occurred during screening colonoscopy happened in colons that had been mechanically well prepared. However, iatrogenic colonic perforation by colonoscopy is a fatal complication for patients with a high anesthetic risk. Mai et al. reported a $40 \%$ mortality rate after iatrogenic colonic perforation in patients with ASA scores of 3 or 4 [27].

The median postoperative hospital stay was 8.5 (range: 5-15) days in this study. According to the time to tolerance of a regular diet, which was a median of 5 (range: 3-6) days, most patients were able to be discharged within one week after surgery. However, Korean patients are usually discharged from the hospital to their home after they can tolerate a regular diet and experience a normal bowel movement, and they tend to settle the legal problem of a medical dispute regarding an iatrogenic complication during their hospital stay. Therefore, the total hospital stay in this study may be longer than that in other countries.

\section{Conclusions}

In iatrogenic colonic perforation during screening colonoscopy, laparoscopic repair should be considered first if there is an experienced laparoscopic surgeon on hand. Laparoscopic repair using an endo- 
scopic linear stapler may be a safe, easy, and effective surgical technique in these cases. Laparoscopic repair using an endoscopic linear stapler could be one of the best surgical procedures to treat colonic perforation related to the performance of screening colonoscopy.

\section{Conflict of interest}

The authors declare no conflict of interest.

\section{References}

1. Matyja M, Pasternak A, Szura M, et al. Cecal intubation rates in different eras of endoscopic technological development. Videosurgery Miniinv 2018; 13: 67-73.

2. Iqbal CW, Chun YS, Farley DR. Colonoscopic perforations: a retrospective review. J Gastrointest Surg 2005; 9: 1229-35.

3. Luning TH, Keemers-Gels ME, Barendregt WB, et al. Colonoscopic perforations: a review of 30,366 patients. Surg Endosc 2007; 21: 994-7.

4. Tulchinsky H, Madhala-Givon O, Wasserberg N, et al. Incidence and management of colonoscopic perforations: 8 years' experience. World J Gastroenterol 2006; 12: 4211-3.

5. Ko CW, Dominitz JA. Complications of colonoscopy: magnitude and management. Gastrointest Endosc Clin N Am 2010; 20: 659-71.

6. An SB, Shin DW, Kim JY, et al. Decision-making in the management of colonoscopic perforation: a multicentre retrospective study. Surg Endosc 2016; 30: 2914-21.

7. Park JY, Choi PW, Jung SM, et al. The outcomes of management for colonoscopic perforation: a 12-year experience at a single institute. Ann Coloproctol 2016; 32: 175-83.

8. Shin DK, Shin SY, Park CY, et al. Optimal methods for the management of iatrogenic colonoscopic perforation. Clin Endosc 2016; 49: 282-8.

9. Strzałka M, Bobrzyński A. Laparoscopy in the treatment of acute abdominal diseases. Videosurgery Miniinv 2008; 3: 1-9.

10. Schlinkert RT, Rasmussen TE. Laparoscopic repair of colonoscopic perforations of the colon. J Laparoendosc Surg 1994; 4: 51-4.

11. Hayashi K, Urata K, Munakata Y, et al. Laparoscopic closure for perforation of the sigmoid colon by endoscopic linear stapler. Surg Laparosc Endosc 1996; 6: 411-3.

12. Miranda L, Settembre A, Piccolboni D, et al. Iatrogenic colonic perforation: repair using laparoscopic technique. Surg Laparosc Endosc Percutan Tech 2011; 21: 170-4.

13. Park JH, Lim SW, Kim SE, et al. Single port laparoscopic repair of sigmoid colon perforation during colonoscopy. Ann Surg Treat Res 2017; 93: 284-6.

14. Wullstein C, Koppen M, Gross E. Laparoscopic treatment of coIonic perforations related to colonoscopy. Surg Endosc 1999; 13: 484-7.

15. Yamamoto A, Ibusuki K, Koga K, et al. Laparoscopic repair of colonic perforation associated with colonoscopy: use of passing sutures and endoscopic linear stapler. Surg Laparosc Endosc Percutan Tech 2001; 11: 19-21.
16. Zhang YQ, Lu W, Yao LQ, et al. Laparoscopic direct suture of perforation after diagnostic colonoscopy. Int I Colorectal Dis 2013; 28: 1505-9.

17. Zhong W, Qiu C, Liu C, et al. Efficacy of laparoscopic primary repair in the treatment of colonic perforation after colonoscopy: a review of 40,127 patients. Surg Laparosc Endosc Percutan Tech 2016; 26: e105-8.

18. Kim J, Lee GJ, Baek JH, et al. Comparison of the surgical outcomes of laparoscopic versus open surgery for colon perforation during colonoscopy. Ann Surg Treat Res 2014; 87: 139-43.

19. ASGE Standards of Practice Committee, Fisher DA, Maple JT, et al. Complications of colonoscopy. Gastrointest Endosc 2011; 74: 745-52.

20. Kim JS, Kim BW, Kim JI, et al. Endoscopic clip closure versus surgery for the treatment of iatrogenic colon perforations developed during diagnostic colonoscopy: a review of 115,285 patients. Surg Endosc 2013; 27: 501-4.

21. Marucci DD, Shakeshaft AJ, Cartmill JA, et al. Grasper trauma during laparoscopic cholecystectomy. Aust N Z I Surg 2000; 70 : 578-81.

22. Cho SB, Lee WS, Joo YE, et al. Therapeutic options for iatrogenic colon perforation: feasibility of endoscopic clip closure and predictors of the need for early surgery. Surg Endosc 2012; 26: 473-9.

23. Kantsevoy SV, Bitner M, Hajiyeva G, et al. Endoscopic management of colonic perforations: clips versus suturing closure (with videos). Gastrointest Endosc 2016; 84: 487-93.

24. Magdeburg R, Collet P, Post S, et al. Endoclipping of iatrogenic colonic perforation to avoid surgery. Surg Endosc 2008; 22: 1500-4.

25. Trecca A, Gaj F, Gagliardi G. Our experience with endoscopic repair of large colonoscopic perforations and review of the literature. Tech Coloproctol 2008; 12: 315-21.

26. Yang DH, Byeon IS, Lee KH, et al. Is endoscopic closure with clips effective for both diagnostic and therapeutic colonoscopy-associated bowel perforation? Surg Endosc 2010; 24: 1177-85.

27. Mai CM, Wen CC, Wen SH, et al. latrogenic colonic perforation by colonoscopy: a fatal complication for patients with a high anesthetic risk. Int J Colorectal Dis 2010; 25: 449-54.

Received: 18.04.2018, accepted: 24.07.2018. 